ISSN 1392-3196 / e-ISSN 2335-8947

Zemdirbyste-Agriculture, vol. 103, No. 4 (2016), p. 347-354

DOI 10.13080/z-a.2016.103.044

\title{
Tractor traffic and nitrogen fertilization affect the herbage production of the red clover/grass sward
}

\author{
Tomasz GLAB, Andrzej ŻABINSKI, Urszula SADOWSKA \\ Institute of Machinery Exploitation, Ergonomics and Production Processes, \\ University of Agriculture in Krakow \\ Balicka 116 B, 31-149 Krakow, Poland \\ E-mail: rtglab@cyf-kr.edu.pl
}

\begin{abstract}
In recent years, soil compaction caused by agricultural machinery has been recognized as one of the main factors that can lower yields of perennial crops. At the same time changes in grassland fertilization intensity are observed. The objectives of the present study were to evaluate the interaction of different $\mathrm{N}$ fertilization rates and the intensity of tractor passes. This study was conducted as a field experiment located on a sandy loam soil classified as Mollic Fluvisol in Mydlniki near Krakow, Poland over a four-year period (2009-2012). The field experiment was established with four replications in a split-plot design with fertilization as the main plot and tractor traffic as the subplot. Three levels of $\mathrm{N}$ fertilization: untreated (control), 80 and $160 \mathrm{~kg} \mathrm{ha}^{-1} \mathrm{~N}$, and four levels of tractor traffic intensity were applied using the following numbers of tractor passes: no passes (control), two passes, four passes and six passes.

Nitrogen (N) fertilization significantly affected the percentage of only three species - Trifolium pratense, Lolium perenne and Festuca pratensis in the sward. T. pratense was negatively affected by with higher $\mathrm{N}$ rates but two grasses, L. perenne and F. pratensis, showed the opposite response. The tractor traffic significantly affected T. pratense and $L$. perenne occurrence in the swards. The percentage of $T$. pratense in the sward was reduced by tractor traffic, and this space was occupied by L. perenne. Changes in the botanical composition reflected in the herbage production. All investigated grass species increased biomass productivity in direct proportion to $\mathrm{N}$ rates. Only $T$. pratense was negatively affected by nitrogen fertilization and since 2011 it started to disappear from the swards treated with higher nitrogen rates. For all investigated components of the mixture no interaction between treatments, tractor traffic and nitrogen fertilization was observed.
\end{abstract}

Key words: botanical composition, grasses, herbage production, nitrogen fertilization, red clover.

\section{Introduction}

Agricultural production systems tend to increase the number of passes and the loads carried on agricultural vehicles, resulting in a potential for increased soil compaction (Newell-Price et al., 2013). According to the European Union Commission, soil compaction is now recognized as one of the main factors that can decrease crop yields and thus is a serious agricultural problem (COM(2006)231 - EUR-Lex - Europa).

Compaction leads to soil structure degradation, which results in worsening physical properties. Increase in bulk density and soil strength, measured as penetration resistance, are associated with the aforementioned changes. The soil compaction leads to reduction in plant yield including decreased production of perennial forage crops (Głąb, 2008). This yield decline is the consequence of both soil compaction and shoot injury caused by wheel traffic. It is a serious problem for perennial crops, where the soil is subjected to compaction without tillage operation. Soil strength is increased year-after-year and all machine traffic during field operation causes direct damage to plants which are reported to be as important in contributing to decreased plant yield as soil compaction. Moreover, perennial forage crop production demands a great intensity of vehicular activity, especially during crop harvesting operations. On the other hand, it has also been reported that yields of perennial plants were not always reduced by compaction and sometimes were larger in compacted soil than in non-compacted soil. These trends could be attributed to better water and nutrient supply and recovery of the soil pore system (Scott et al., 2005).

In recent years, more powerful and heavier tractors and machinery have been used on farms. On the other hand, the extensification in livestock production is promoted by the European Union and this has resulted in the reduction of the stocking rate and fertilizer application (Regulation (EEC) 2078/92). The design and implementation of the resulting national agri-environmental schemes modified to a great extent the direction of grassland system development in the European Union countries. One of the most important changes was in the areas of regulation and incentives to limit grassland fertilizer application in order to reduce nutrient losses and mitigate soil and water pollution. The second direction in grassland system modification was in the area of maintenance of biodiversity and landscape expressed as species richness (Baritaux et al., 2016).

Grasslands are strongly affected by field management. It has frequently been shown that the 
application of high doses of fertilizers and intensive sward utilization have negative effects on plant species diversity (Tephnadze et al., 2014). Chemical and physical soil characteristics are related not only to natural soil properties but also to fertilization inputs, and influence both species richness and species composition of vascular plants (Marini et al., 2007). Fertilization, especially with nitrogen and phosphorus, can increase dry matter production. However, fertilization may improve not only dry matter productivity, but could also affect botanical composition (Koc, 2013). Large nutrient availability leads to an increase of competitive asymmetry among plant species and to a quick exclusion of short, slowly growing plant species (Gaujour et al., 2012). It has been well established that high-nitrogen fertilizer applications generally decrease species diversity (Klimek et al., 2007). Nitrogen fertilization usually plays a favourable role in the growing conditions of tall grasses like Festuca pratensis, Poa pratensis and Agropyron repens. One of the most important components in the botanical composition of grasslands is legumes. However, the nitrogen fertilization used to increase dry matter yield results in a decrease in legume ratio in the botanical composition (Głąb, Kacorzyk, 2011; Koc, 2013). The question is whether there is an interaction between traffic intensity and level of fertilization in terms of plant growth conditions. However, the interaction between the effect of soil compaction and the effect of fertilization used on grasslands has not been studied thoroughly so far. This investigation is based on the Strategy for Soil Protection (Regulation (EEC) 2078/92) with the aim of filling the gap in knowledge about soil and its environmental and productive functions.

In this study, it was hypothesized that (i) tractor traffic affects biomass production on grasslands and (ii) nitrogen fertilization results in changes in herbage production and botanical composition of swards. The aim of this investigation was to study the interaction of different nitrogen fertilization rates and traffic intensity on grass/red clover mixture with the focus on herbage production and botanical composition of swards.

\section{Material and methods}

Site, location, and climate. This study was conducted as a field experiment located in Mydlniki near Krakow, Poland $\left(50^{\circ} 04^{\prime} \mathrm{N}, 19^{\circ} 51^{\prime} \mathrm{E}, 211 \mathrm{~m}\right.$ a.s.l., slope $2^{\circ}$ ) over a four-year period (2009-2012). The field experiment was carried out on sandy loam Mollic Fluvisol soil (WRB, 2014). Table 1 details some of the soil characteristics.

The climate of the experimental site is temperatecontinental. The average annual precipitation reaches $681 \mathrm{~mm}$ per year and during the studied period varied from 552 in 2011 to $1010 \mathrm{~mm}$ in 2010 . The mean daily temperature is $7.4^{\circ} \mathrm{C}$ and varied from $7.9^{\circ} \mathrm{C}$ in 2010 to $8.7^{\circ} \mathrm{C}$ in 2011.

Field trial design and treatments. The soil before compaction was ploughed in the autumn of 2008 and harrowed in March 2009 for seedbed preparation then seeds of red clover/grass mixture were sown. Experimental plots $\left(9 \mathrm{~m}^{2}\right)$ were established with four replications in a split-plot design with fertilization as the main plot and tractor traffic as the subplot. Each plot was sown with a mixture of $10 \mathrm{~kg} \mathrm{ha}^{-1}$ of perennial ryegrass (Lolium perenne L.) cv. 'Diament', $13 \mathrm{~kg} \mathrm{ha}^{-1}$ of meadow fescue (Festuca pratensis Huds.) cv. 'Skra', $3 \mathrm{~kg} \mathrm{ha}^{-1}$ of timothy (Phleum pratense L.) cv. 'Skala', $2 \mathrm{~kg} \mathrm{ha}^{-1}$ of Kentucky bluegrass (Poa pratensis L.) cv. 'Skiz' and $2 \mathrm{~kg} \mathrm{ha}^{-1}$ of red clover (Trifolium pratense L.) cv. 'Nike'.
Table 1. Basic soil physical and chemical properties of Mollic Fluvisol (0-20 cm layer) from the trial location

\begin{tabular}{ccc}
\hline Parameter & Unit & Value \\
\hline $\mathrm{pH}(\mathrm{KCl})$ & & 6.5 \\
Organic C & $\mathrm{g} \mathrm{kg}^{-1}$ & 12.5 \\
Total N & $\mathrm{g} \mathrm{kg}^{-1}$ & 1.39 \\
C:N ratio & & 9.0 \\
$\mathrm{P}$ & $\mathrm{mg} \mathrm{kg}^{-1}$ & 107.2 \\
$\mathrm{~K}$ & $\mathrm{mg} \mathrm{kg}^{-1}$ & 138.0 \\
$\mathrm{Mg}$ & $\mathrm{mg} \mathrm{kg}^{-1}$ & 67.9 \\
Solid particle density & $\mathrm{Mg} \mathrm{m}^{-3}$ & 2.65 \\
Sand & $\mathrm{g} \mathrm{kg}^{-1}$ & 560 \\
Silt & $\mathrm{g} \mathrm{kg}^{-1}$ & 270 \\
Clay & $\mathrm{g} \mathrm{kg}^{-1}$ & 170 \\
Texture & & sandy loam \\
\hline
\end{tabular}

The nitrogen fertilizer (ammonium nitrate, 34\% N) treatments used were: untreated (control, N0), $80 \mathrm{~kg}$ $\mathrm{ha}^{-1} \mathrm{~N}(\mathrm{~N} 80)$ and $160 \mathrm{~kg} \mathrm{ha}^{-1} \mathrm{~N}$ (N160). Doses of nitrogen were applied three times a year, in March and after the first and second harvest (in May and July, respectively) in proportions of 50, 25 and $25 \%$, respectively. Phosphorus and potassium fertilization was always of the value $40 \mathrm{~kg}$ ha-1 $\mathrm{P}_{2} \mathrm{O}_{5}$ (triple super phosphate, $46 \% \mathrm{P}_{2} \mathrm{O}_{5}$ ) and $80 \mathrm{~kg} \mathrm{~K}_{2} \mathrm{O}$ (potassium chloride, $60 \% \mathrm{~K}_{2} \mathrm{O}$ ). These doses were applied every year in March and after the second harvest. No organic fertilization was applied on the experimental plots.

Four tractor passes treatments were investigated: no passes (control, P0), two passes (P2), four passes (P4) and six passes (P6). Tractor Ursus C-360 (Ursus Ltd., Poland) of $2056 \mathrm{~kg}$ weight was used for traffic simulation. The inflation pressure of the front tires (6.00-16 6PR) of the tractor was $150 \mathrm{kPa}$ and that of rear tires (14.9-28 $8 \mathrm{PR}) 100 \mathrm{kPa}$. Multiple passes were applied in a wheelbeside-wheel design, after every harvest (three times a year). The traffic intensity was applied at a soil moisture content of approximately $0.30 \mathrm{~cm}^{-3} \mathrm{~cm}^{-3}\left( \pm 0.03 \mathrm{~cm}^{-3} \mathrm{~cm}^{-3}\right)$. This water content corresponded to the value of the field water capacity.

Measurements. Starting from the following year after sowing, plots were cut at a stubble height of $5 \mathrm{~cm}$ three times a year with the first cut being at the moment when approximately $20 \%$ of legumes were flowering and the other two cuts done when grasses began to flower. Dry matter (DM) production was determined by cutting each plot using a meadow mower Agria (Agria-Werke GmbH, Germany). Fresh herbage yield was recorded in the field. Herbage samples were taken from each plot and dried for 48 hours at $80^{\circ} \mathrm{C}$ to calculate the dry matter yield. Botanical composition was based on weight contribution of a particular species in total sample weight. For all kinds of cuts and at all treatments, the central $1 \mathrm{~m}^{2}$ of the plot was cut with shears to a height of $5 \mathrm{~cm}$. The samples were separated according to their species and dried to a constant weight at $80^{\circ} \mathrm{C}$.

Statistical analysis. An analysis of variance for a split-plot design was performed to evaluate the significance of traffic intensity and nitrogen fertilization on the botanical composition and biomass production using the statistical software package STATISTICA 10.0 (StatSoft Inc., USA). Means were compared using Tukey's test with a level of significance of $P<0.05$.

\section{Results}

Botanical composition. In the first year of the experiment, before the treatments were applied, the proportion between the species was as follows: 
T. pratense $18.2 \%$, L. perenne $37.1 \%$, F. pratensis $7.4 \%$, P. pratensis $7.4 \%$, and $P h$. pratense $14.8 \%$ (Fig.). Other grasses and forbs together accounted for $15.0 \%$. Forbs were mainly represented by Plantago major, Conyza canadensis, Capsella bursa-pastoris, Taraxacum officinale, Chenopodium album and Cichorium intybus. Other grasses like Poa annua and Echinochloa crusgalli appeared mainly during the period 2009-2010 and subsequently disappeared from the swards.

The botanical composition of swards changed during the four years of the experiment. The participation of two of the tested species, T. pratense and Ph. pratense, in the sward decreased. In 2012, the percentage of T. pratense was only $1.6 \%$ and $P h$. pratense $-7.2 \%$. The contribution of $L$. perenne ( $35.0 \%$ in average) was at the same level during the entire duration of the experiment. Two species, $P$. pratensis and $F$. pratensis, increased their participation up to $36.0 \%$ and $12.8 \%$, respectively. According to the analysis of variance both factors, tractor passes and nitrogen fertilization, play a significant role in the botanical composition of swards. Furthermore the interaction between the year and the fertilization was observed.

Nitrogen fertilization significantly affected the percentage of only three species, T. pratense, L. perenne and $F$. pratensis. $T$. pratense was negatively correlated with higher nitrogen rates and it varied from $13.4 \%$ at the N0 treatment to $5.4 \%$ at the N160 treatment. Two grasses
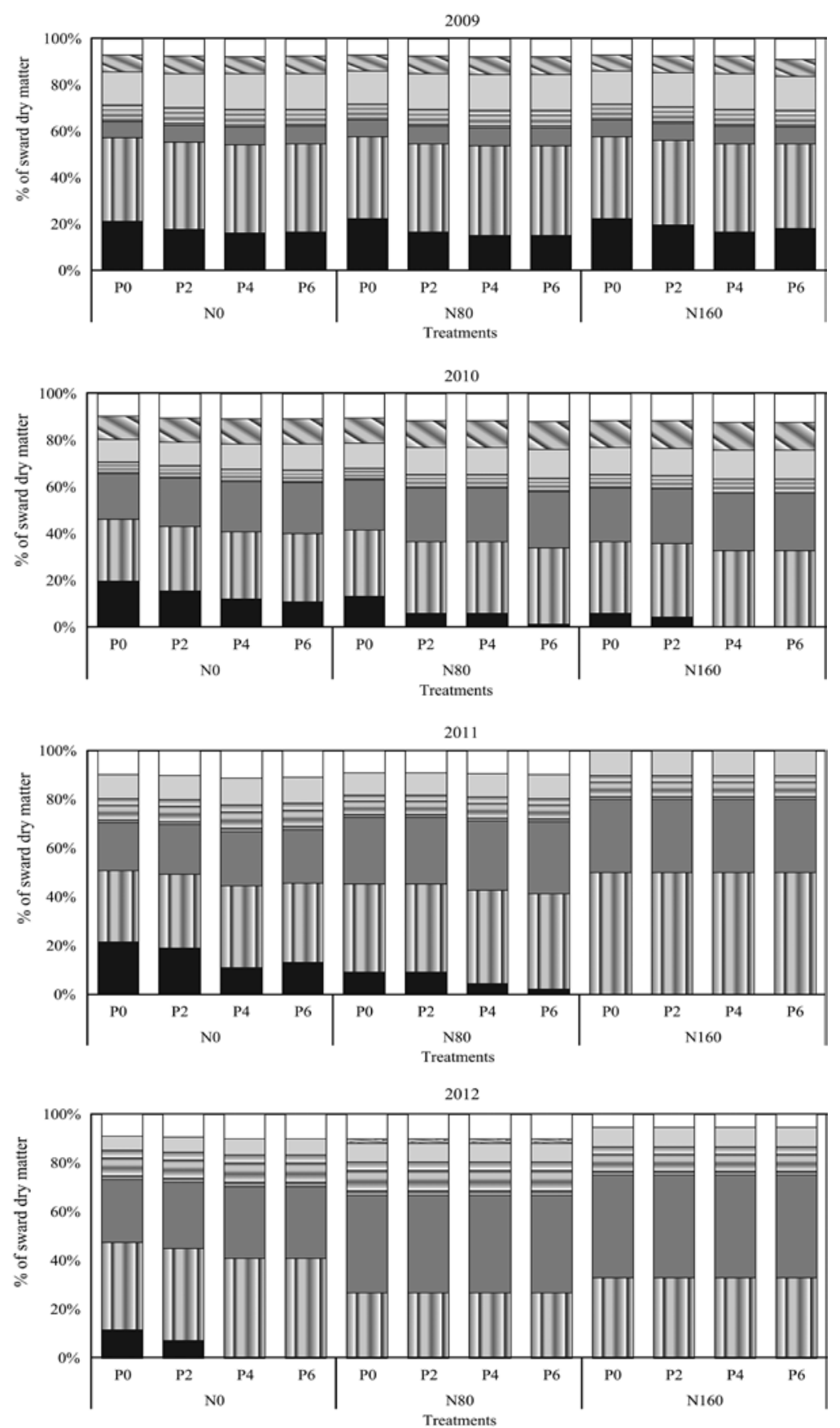

P0 - untreated (control), P2 - two tractor passes, P4 - four tractor passes, P6 - six tractor passes; N0 - unfertilized (control), N80 - fertilization rate of $80 \mathrm{~kg} \mathrm{ha}^{-1} \mathrm{~N}, \mathrm{~N} 160$ - fertilization rate of $160 \mathrm{~kg} \mathrm{ha}^{-1} \mathrm{~N} ; \mathrm{TrPr}-$ Trifolium pratense, LoPe - Lolium perenne, $\mathrm{FePr}-$ Festuca pratensis, $\mathrm{PoPr}-$ Poa pratensis, $\mathrm{PhPr}$ - Phleum pratensis, $\mathrm{Gr}$ - other grasses, $\mathrm{Fo}$ - forbs

Figure. Effect of tractor traffic and nitrogen fertilization on the botanical composition of swards 
showed the opposite reaction. $F$. pratensis percentage increased from $19.3 \%$ at the N0 treatment to $25.8 \%$ at the N160 treatment. For L. perenne, the statistically higher percentage was at the N160 treatment $(37.9 \%)$ when compared with the N0 and N80 treatments. Both species, $P h$. pratense and $P$. pratensis, were not affected by $\mathrm{N}$ fertilization. Two species, T. pratense and L. perenne, showed significant interaction between fertilization and the year of the experiment. The differences in the reaction of these two species to $\mathrm{N}$ fertilization appeared in 2009. The participation of $T$. pratense in the swards increased in the first year of the trial, when higher nitrogen rates were applied, whereas in the following years, the reaction was just the opposite. The percentage of $L$. perenne decreased in 2009 from $37.2 \%$ at the N0 to $36.2 \%$ at the N160. The following year, this species positively reacted to nitrogen fertilization.

The tractor traffic significantly affected the participation of $T$. pratense and L. perenne in the sward biomass. The percentage of $T$. pratense was reduced by wheel traffic, and this space was occupied by L. perenne. At the $\mathrm{P} 0$ treatment the participation of $T$. pratense was $12.3 \%$ and it decreased to $6.5 \%$ at the P 6 treatment. Other tested grasses were not influenced by tractor traffic.

Dry matter production. The mean annual DM yield of red clover/grass mixture was $10.52 \mathrm{t} \mathrm{ha}^{-1}$ DM. The annual biomass production of the tested mixture was affected by the experimental treatments, nitrogen fertilization and soil tractor traffic (Tables 2-6). Fertilization significantly increased plant yields. The lower value for dry matter was obtained at the N0 treatment, 8.38 tha $^{-1}$ DM in average during the 2009-2012 period. For higher nitrogen rates the yields increased and were 10.20 and $12.28 \mathrm{t} \mathrm{ha}^{-1} \mathrm{DM}$ for the N80 and N160, respectively. A similar relationship was observed during every year of the trial. Soil compaction caused by tractor wheeling reduced yields from $11.11 \mathrm{tha}^{-1} \mathrm{DM}$ at the $\mathrm{P} 0$ to $9.74 \mathrm{tha}^{-1} \mathrm{DM}$ at the P6. This relationship was maintained during the whole experimental period.

The rule that fertilization increased yield of grassland species mixture was not always followed by its individual components. All species were significantly affected by nitrogen fertilization. However, for some species, this effect was different during particular years. Only two species, $T$. pratense and $L$. perenne, were sensitive to tractor wheeling. The highest annual dry matter yield was obtained for L. perenne $\left(3.68 \mathrm{tha}^{-1} \mathrm{DM}\right.$ in average). These values for other grasses $F$. pratensis, Ph. pratense and P. pratensis were $2.48,1.14$ and 0.94 $\mathrm{t} \mathrm{ha}^{-1} \mathrm{DM}$, respectively. $T$. pratense was characterized by the lowest dry matter production $\left(0.90 \mathrm{t} \mathrm{ha}^{-1} \mathrm{DM}\right)$. The variation in herbage production of tested species was observed during the four years of the experiment. Three of them, T. pratense, L. perenne and Ph. pratense, decreased productivity. However, two others increased dry matter yield during this period. Consequently, the total annual yield of the sward was at the same level from 2009 to 2012.

All species were affected by nitrogen fertilization. However, this reaction changed with time. In the first year of the trial, i.e. 2009, T. pratense increased the dry matter productivity in direct proportion to nitrogen rates. This relationship was observed for all three cuts. Since 2010, this species started to disappear from swards treated with higher N rates. Starting from 2011, during the time of the first cut, there were not any plants of T. pratense at the N160 treatment. Other investigated grass species increased productivity when higher $\mathrm{N}$ rates were applied. The two other grass components of the mixture, $L$. perenne and F. pratensis, showed interaction with the year. The highest annual herbage productivity of $L$. perenne was recorded at the N160 treatment (6.16 tha ${ }^{-1}$ DM) in 2011. This productivity was significantly lower in other years. The annual yields of $F$. pratensis increased specifically during the years of the experiment. However, this effect was found to be higher when treated with higher nitrogen rates.

The most susceptible species to tractor traffic was T. pratense. The significant decrease in DM production was noticed during all years and for all cuts. The highest mean annual yield was obtained at the P0 treatment $\left(1.30 \mathrm{t} \mathrm{ha}^{-1} \mathrm{DM}\right)$ and the lower mean annual yield at the P6 (0.61 t ha $\left.{ }^{-1} \mathrm{DM}\right)$. Multiple tractor passes slowly eliminated this species and finally it disappeared at the P4 and P6 in 2012. The grasses showed a negative reaction

Table 2. Effect of tractor traffic and nitrogen fertilization on herbage yield ( $\mathrm{t} \mathrm{ha}^{-1} \mathrm{DM}$ ) of Trifolium pratense

\begin{tabular}{|c|c|c|c|c|c|c|c|c|c|c|c|c|c|}
\hline \multirow{2}{*}{\multicolumn{2}{|c|}{ Treatments }} & \multicolumn{3}{|c|}{2009} & \multicolumn{3}{|c|}{2010} & \multicolumn{3}{|c|}{2011} & \multicolumn{3}{|c|}{2012} \\
\hline & & $1^{\text {st }}$ cut & $2^{\text {nd }}$ cut & $3^{\text {rd }}$ cut & $1^{\text {st }} \mathrm{cut}$ & $2^{\text {nd }}$ cut & $3^{\text {rd }}$ cut & $1^{\text {st }}$ cut & $2^{\text {nd }}$ cut & $3^{\text {rd }}$ cut & $1^{\text {st }}$ cut & $2^{\text {nd }}$ cut & $3^{\text {rd }}$ cut \\
\hline \multirow{4}{*}{ N0 } & $\mathrm{P} 0$ & 0.86 & 0.46 & 0.45 & 0.69 & 0.47 & 0.46 & $0.87 \mathrm{a}$ & 0.66 & $0.65 \mathrm{a}$ & $0.35 \mathrm{a}$ & $0.33 \mathrm{a}$ & $0.25 \mathrm{a}$ \\
\hline & P2 & 0.76 & 0.40 & 0.38 & 0.56 & 0.44 & 0.37 & $0.71 \mathrm{a}$ & 0.60 & $0.48 \mathrm{a}$ & $0.28 \mathrm{a}$ & $0.17 \mathrm{~b}$ & $0.13 \mathrm{~b}$ \\
\hline & P4 & 0.73 & 0.37 & 0.31 & 0.44 & 0.38 & 0.29 & $0.39 \mathrm{bc}$ & 0.36 & $0.26 \mathrm{bc}$ & $0.00 \mathrm{~b}$ & $0.00 \mathrm{c}$ & $0.00 \mathrm{c}$ \\
\hline & P6 & 0.71 & 0.33 & 0.22 & 0.35 & 0.32 & 0.22 & $0.49 \mathrm{~b}$ & 0.39 & $0.31 \mathrm{~b}$ & $0.00 \mathrm{~b}$ & $0.00 \mathrm{c}$ & $0.00 \mathrm{c}$ \\
\hline \multirow{4}{*}{ N80 } & P0 & 1.13 & 0.66 & 0.74 & 0.83 & 0.41 & 0.39 & $0.45 \mathrm{~b}$ & 0.32 & $0.31 \mathrm{~b}$ & $0.00 \mathrm{~b}$ & $0.00 \mathrm{c}$ & $0.00 \mathrm{c}$ \\
\hline & P2 & 0.91 & 0.51 & 0.50 & 0.34 & 0.18 & 0.13 & $0.43 \mathrm{~b}$ & 0.33 & $0.28 \mathrm{~b}$ & $0.00 \mathrm{~b}$ & $0.00 \mathrm{c}$ & $0.00 \mathrm{c}$ \\
\hline & P4 & 0.84 & 0.44 & 0.38 & 0.30 & 0.18 & 0.13 & $0.20 \mathrm{~cd}$ & 0.15 & $0.11 \mathrm{~cd}$ & $0.00 \mathrm{~b}$ & $0.00 \mathrm{c}$ & $0.00 \mathrm{c}$ \\
\hline & P6 & 0.78 & 0.40 & 0.31 & 0.08 & 0.05 & 0.03 & $0.08 \mathrm{~d}$ & 0.07 & $0.05 \mathrm{~d}$ & $0.00 \mathrm{~b}$ & $0.00 \mathrm{c}$ & $0.00 \mathrm{c}$ \\
\hline \multirow{4}{*}{ N160 } & P0 & 1.38 & 0.81 & 0.93 & 0.38 & 0.21 & 0.16 & $0.00 \mathrm{~d}$ & 0.00 & $0.00 \mathrm{~d}$ & $0.00 \mathrm{~b}$ & $0.00 \mathrm{c}$ & $0.00 \mathrm{c}$ \\
\hline & P2 & 1.14 & 0.66 & 0.71 & 0.31 & 0.18 & 0.13 & $0.00 \mathrm{~d}$ & 0.00 & $0.00 \mathrm{~d}$ & $0.00 \mathrm{~b}$ & $0.00 \mathrm{c}$ & $0.00 \mathrm{c}$ \\
\hline & P4 & 0.97 & 0.50 & 0.45 & 0.00 & 0.00 & 0.00 & $0.00 \mathrm{~d}$ & 0.00 & $0.00 \mathrm{~d}$ & $0.00 \mathrm{~b}$ & $0.00 \mathrm{c}$ & $0.00 \mathrm{c}$ \\
\hline & P6 & 1.04 & 0.54 & 0.50 & 0.00 & 0.00 & 0.00 & $0.00 \mathrm{~d}$ & 0.00 & $0.00 \mathrm{~d}$ & $0.00 \mathrm{~b}$ & $0.00 \mathrm{c}$ & $0.00 \mathrm{c}$ \\
\hline \multicolumn{14}{|c|}{ Means for fertilization } \\
\hline & N0 & $0.76 \mathrm{~b}$ & $0.39 \mathrm{~b}$ & $0.34 \mathrm{~b}$ & $0.51 \mathrm{a}$ & $0.40 \mathrm{a}$ & $0.33 \mathrm{a}$ & $0.62 \mathrm{a}$ & $0.50 \mathrm{a}$ & $0.43 \mathrm{a}$ & $0.16 \mathrm{a}$ & $0.13 \mathrm{a}$ & $0.10 \mathrm{a}$ \\
\hline & N80 & $0.92 \mathrm{~b}$ & $0.50 \mathrm{a}$ & $0.48 \mathrm{a}$ & $0.39 \mathrm{~b}$ & $0.21 \mathrm{~b}$ & $0.17 \mathrm{~b}$ & $0.29 \mathrm{~b}$ & $0.22 \mathrm{~b}$ & $0.19 \mathrm{~b}$ & $0.00 \mathrm{~b}$ & $0.00 \mathrm{~b}$ & $0.00 \mathrm{~b}$ \\
\hline & N160 & $1.13 \mathrm{a}$ & $0.63 \mathrm{a}$ & $0.64 \mathrm{a}$ & $0.17 \mathrm{c}$ & $0.10 \mathrm{c}$ & $0.07 \mathrm{c}$ & $0.00 \mathrm{c}$ & $0.00 \mathrm{c}$ & $0.00 \mathrm{c}$ & $0.00 \mathrm{~b}$ & $0.00 \mathrm{~b}$ & $0.00 \mathrm{~b}$ \\
\hline \multicolumn{14}{|c|}{ Means for tractor passes } \\
\hline & P0 & 1.12 & 0.65 & $0.70 \mathrm{a}$ & $0.63 \mathrm{a}$ & $0.37 \mathrm{a}$ & $0.34 \mathrm{a}$ & $0.44 \mathrm{a}$ & $0.32 \mathrm{a}$ & $0.32 \mathrm{a}$ & $0.12 \mathrm{a}$ & $0.11 \mathrm{a}$ & $0.08 \mathrm{a}$ \\
\hline & P2 & 0.94 & 0.52 & $0.53 \mathrm{ab}$ & $0.41 \mathrm{~b}$ & $0.27 \mathrm{~b}$ & $0.21 \mathrm{~b}$ & $0.38 \mathrm{a}$ & $0.31 \mathrm{a}$ & $0.25 \mathrm{a}$ & $0.09 \mathrm{a}$ & $0.06 \mathrm{~b}$ & $0.04 \mathrm{~b}$ \\
\hline & P4 & 0.85 & 0.44 & $0.38 \mathrm{~b}$ & $0.24 \mathrm{c}$ & $0.19 \mathrm{c}$ & $0.14 \mathrm{c}$ & $0.19 \mathrm{~b}$ & $0.17 \mathrm{~b}$ & $0.12 \mathrm{~b}$ & $0.00 \mathrm{~b}$ & $0.00 \mathrm{~b}$ & $0.00 \mathrm{c}$ \\
\hline & P6 & 0.84 & 0.42 & $0.34 \mathrm{~b}$ & $0.14 \mathrm{c}$ & $0.12 \mathrm{c}$ & $0.08 \mathrm{c}$ & $0.19 \mathrm{~b}$ & $0.15 \mathrm{~b}$ & $0.12 \mathrm{~b}$ & $0.00 \mathrm{~b}$ & $0.00 \mathrm{~b}$ & $0.00 \mathrm{c}$ \\
\hline
\end{tabular}

Notes. N0 - unfertilized (control), N80 - fertilization rate of $80 \mathrm{~kg} \mathrm{ha}^{-1} \mathrm{~N}, \mathrm{~N} 160$ - fertilization rate of $160 \mathrm{~kg} \mathrm{ha}^{-1} \mathrm{~N}$; P0 - untreated (control), P2 - two tractor passes, P4 - four tractor passes, P6 - six tractor passes. For each column, mean values with different superscripts are significantly different $(P<0.05)$, superscripts used only for significant differences according to ANOVA, means without superscripts are not significantly different. 
Table 3. Effect of tractor traffic and nitrogen fertilization on herbage yield ( $\left.\mathrm{t} \mathrm{ha}^{-1} \mathrm{DM}\right)$ of Lolium perenne

\begin{tabular}{|c|c|c|c|c|c|c|c|c|c|c|c|c|c|}
\hline \multirow{2}{*}{\multicolumn{2}{|c|}{ Treatments }} & \multicolumn{3}{|c|}{2009} & \multicolumn{3}{|c|}{2010} & \multicolumn{3}{|c|}{2011} & \multicolumn{3}{|c|}{2012} \\
\hline & & $1^{\text {st }} \mathrm{cut}$ & $2^{\text {nd }}$ cut & $3^{\text {rd }}$ cut & $1^{\text {st }} \mathrm{cut}$ & $2^{\text {nd }}$ cut & $3^{\text {rd }}$ cut & $1^{\text {st }}$ cut & $2^{\text {nd }} \mathrm{cut}$ & $3^{\text {rd }}$ cut & $1^{\text {st }}$ cut & $2^{\text {nd }}$ cut & $3^{\text {rd }}$ cut \\
\hline \multirow{4}{*}{ N0 } & P0 & 1.44 & 0.77 & 0.74 & 0.93 & 0.64 & 0.62 & 1.22 & 0.89 & 0.88 & 1.09 & 1.03 & 0.77 \\
\hline & P2 & 1.45 & 0.76 & 0.69 & 1.01 & 0.78 & 0.66 & 1.22 & 1.01 & 0.77 & 1.36 & 0.93 & 0.71 \\
\hline & P4 & 1.54 & 0.76 & 0.61 & $1 \cdot 08$ & 0.92 & 0.70 & 1.16 & 1.09 & 0.78 & 1.33 & 0.91 & 0.73 \\
\hline & P6 & 1.53 & 0.72 & 0.48 & 0.94 & 0.86 & 0.59 & 1.23 & 0.92 & 0.75 & 1.21 & 0.85 & 0.71 \\
\hline \multirow{4}{*}{ N80 } & P0 & 1.77 & 1.02 & 1.10 & 1.73 & 0.88 & 0.83 & 1.78 & 1.26 & 1.22 & 1.50 & 0.96 & 0.79 \\
\hline & P2 & 1.99 & 1.10 & 1.06 & 1.85 & 1.00 & 0.68 & 1.71 & 1.33 & 1.12 & 1.60 & 0.94 & 0.71 \\
\hline & P4 & 2.02 & 1.06 & 0.92 & 1.61 & 0.97 & 0.70 & 1.68 & 1.21 & 1.07 & 1.46 & 0.70 & 0.59 \\
\hline & P6 & 1.96 & 1.00 & 0.76 & 1.76 & 0.89 & 0.59 & 1.61 & 1.24 & 0.96 & 1.43 & 0.76 & 0.58 \\
\hline \multirow{4}{*}{ N160 } & P0 & 2.13 & 1.25 & 1.42 & 2.03 & 1.16 & 0.89 & 2.75 & 1.85 & 1.70 & 1.87 & 1.20 & 0.90 \\
\hline & $\mathrm{P} 2$ & 2.12 & 1.19 & 1.24 & 2.15 & 1.22 & 0.94 & 2.79 & 1.91 & 1.51 & 1.97 & 1.04 & 1.02 \\
\hline & P4 & 2.21 & 1.13 & 0.96 & 2.07 & 1.20 & 0.87 & 2.86 & 1.83 & 1.59 & 2.02 & 0.98 & 0.85 \\
\hline & P6 & 2.11 & 1.11 & 0.99 & 2.15 & 1.02 & 0.73 & 2.57 & 1.87 & 1.38 & 1.98 & 0.91 & 0.72 \\
\hline \multicolumn{14}{|c|}{ Means for fertilization } \\
\hline & N0 & $1.49 \mathrm{~b}$ & $0.76 \mathrm{~b}$ & $0.63 \mathrm{~b}$ & $0.99 \mathrm{~b}$ & $0.80 \mathrm{~b}$ & $0.64 \mathrm{a}$ & $1.21 \mathrm{~b}$ & $0.98 \mathrm{~b}$ & $0.80 \mathrm{~b}$ & $1.25 \mathrm{~b}$ & 0.93 & $0.73 \mathrm{ab}$ \\
\hline & N80 & $1.94 \mathrm{a}$ & $1.04 \mathrm{a}$ & $0.96 \mathrm{a}$ & $1.74 \mathrm{a}$ & $0.94 \mathrm{a}$ & $0.70 \mathrm{~b}$ & $1.70 \mathrm{~b}$ & $1.26 \mathrm{~b}$ & $1.09 \mathrm{~b}$ & $1.50 \mathrm{ab}$ & 0.84 & $0.67 \mathrm{~b}$ \\
\hline & N160 & $2.14 \mathrm{a}$ & $1.17 \mathrm{a}$ & $1.15 \mathrm{a}$ & $2.10 \mathrm{a}$ & $1.15 \mathrm{a}$ & $0.86 \mathrm{a}$ & $2.74 \mathrm{a}$ & $1.87 \mathrm{a}$ & $1.55 \mathrm{a}$ & $1.96 \mathrm{a}$ & 1.03 & $0.87 \mathrm{a}$ \\
\hline \multicolumn{14}{|c|}{ Means for tractor passes } \\
\hline & $\overline{\mathrm{P} 0}$ & 1.78 & 1.01 & $1.09 \mathrm{a}$ & 1.57 & 0.89 & 0.78 & 1.92 & 1.33 & $1.27 \mathrm{a}$ & 1.49 & 1.06 & 0.82 \\
\hline & $\mathrm{P} 2$ & 1.85 & 1.02 & $1.00 \mathrm{a}$ & 1.67 & 1.00 & 0.76 & 1.91 & 1.42 & $1.13 \mathrm{ab}$ & 1.65 & 0.97 & 0.82 \\
\hline & P4 & 1.93 & 0.98 & $0.83 \mathrm{ab}$ & 1.59 & 1.03 & 0.76 & 1.90 & 1.37 & $1.15 \mathrm{ab}$ & 1.60 & 0.86 & 0.72 \\
\hline & P6 & 1.87 & 0.94 & $0.74 \mathrm{~b}$ & 1.62 & 0.92 & 0.64 & 1.81 & 1.34 & $1.03 \mathrm{~b}$ & 1.54 & 0.84 & 0.67 \\
\hline
\end{tabular}

Explanations under Table 2

Table 4. Effect of tractor traffic and nitrogen fertilization on herbage yield ( $\mathrm{t}$ ha $\left.{ }^{-1} \mathrm{DM}\right)$ of Festuca pratensis

\begin{tabular}{|c|c|c|c|c|c|c|c|c|c|c|c|c|c|}
\hline \multirow{2}{*}{\multicolumn{2}{|c|}{ Treatments }} & \multicolumn{3}{|c|}{2009} & \multicolumn{3}{|c|}{2010} & \multicolumn{3}{|c|}{2011} & \multicolumn{3}{|c|}{2012} \\
\hline & & $1^{\text {st }}$ cut & $2^{\text {nd }}$ cut & $3^{\text {rd }}$ cut & $1^{\text {st }} \mathrm{cut}$ & $2^{\text {nd }}$ cut & $3^{\text {rd }}$ cut & $1^{\text {st }} \mathrm{cut}$ & $2^{\text {nd }}$ cut & $3^{\text {rd }}$ cut & $1^{\text {st }}$ cut & $2^{\text {nd }}$ cut & $3^{\text {rd }}$ cut \\
\hline \multirow{4}{*}{ N0 } & P0 & 0.29 & 0.15 & 0.15 & 0.69 & 0.47 & 0.46 & 0.81 & 0.59 & 0.59 & 0.81 & 0.76 & 0.56 \\
\hline & P2 & 0.29 & 0.15 & 0.14 & 0.75 & 0.58 & 0.49 & 0.81 & 0.67 & 0.51 & 0.95 & 0.66 & 0.53 \\
\hline & P4 & 0.31 & 0.15 & 0.12 & 0.80 & 0.68 & 0.52 & 0.78 & 0.72 & 0.52 & 0.97 & 0.64 & 0.55 \\
\hline & P6 & 0.31 & 0.14 & 0.10 & 0.70 & 0.64 & 0.44 & 0.82 & 0.61 & 0.50 & 0.89 & 0.58 & 0.52 \\
\hline \multirow{4}{*}{ N80 } & P0 & 0.35 & 0.20 & 0.22 & 1.28 & 0.65 & 0.61 & 1.34 & 0.95 & 0.92 & 2.20 & 1.41 & 1.13 \\
\hline & P2 & 0.40 & 0.22 & 0.21 & 1.37 & 0.74 & 0.50 & 1.28 & 1.00 & 0.84 & 2.41 & 1.39 & 1.05 \\
\hline & P4 & 0.40 & 0.21 & 0.18 & 1.20 & 0.72 & 0.52 & 1.26 & 0.91 & 0.80 & 2.14 & 1.04 & 0.86 \\
\hline & P6 & 0.39 & 0.20 & 0.15 & 1.31 & 0.66 & 0.44 & 1.21 & 0.93 & 0.72 & 2.07 & 1.12 & 0.86 \\
\hline \multirow{4}{*}{ N160 } & P0 & 0.43 & 0.25 & 0.28 & 1.50 & $0 \cdot 86$ & 0.66 & 1.65 & 1.11 & 1.02 & 2.49 & 1.50 & 1.11 \\
\hline & $\mathrm{P} 2$ & 0.42 & 0.24 & 0.25 & 1.59 & 0.91 & 0.70 & 1.67 & 1.15 & 0.91 & 2.61 & 1.30 & 1.25 \\
\hline & P4 & 0.44 & 0.23 & 0.19 & 1.53 & 0.89 & 0.65 & 1.72 & 1.10 & 0.96 & 2.53 & 1.24 & 1.04 \\
\hline & P6 & 0.42 & 0.22 & 0.20 & 1.59 & 0.76 & 0.54 & 1.54 & 1.12 & 0.83 & 2.52 & 1.17 & 0.87 \\
\hline \multicolumn{14}{|c|}{ Means for fertilization } \\
\hline & N0 & $0.30 \mathrm{~b}$ & $0.15 \mathrm{~b}$ & $0.13 \mathrm{~b}$ & $0.73 \mathrm{~b}$ & $0.59 \mathrm{~b}$ & $0.48 \mathrm{~b}$ & $0.81 \mathrm{~b}$ & $0.65 \mathrm{~b}$ & $0.53 \mathrm{~b}$ & $0.90 \mathrm{~b}$ & $0.66 \mathrm{~b}$ & $0.54 \mathrm{~b}$ \\
\hline & N80 & $0.39 \mathrm{ab}$ & $0.21 \mathrm{a}$ & $0.19 \mathrm{ab}$ & $1.29 \mathrm{ab}$ & $0.69 \mathrm{ab}$ & $0.52 \mathrm{ab}$ & $1.27 \mathrm{ab}$ & $0.94 \mathrm{ab}$ & $0.82 \mathrm{a}$ & $2.21 \mathrm{a}$ & $1.24 \mathrm{a}$ & $0.97 \mathrm{a}$ \\
\hline & N160 & $0.43 \mathrm{a}$ & $0.23 \mathrm{a}$ & $0.23 \mathrm{a}$ & $1.56 \mathrm{a}$ & $0.85 \mathrm{a}$ & $0.64 \mathrm{a}$ & $1.65 \mathrm{a}$ & $1.12 \mathrm{a}$ & $0.93 \mathrm{a}$ & $2.54 \mathrm{a}$ & $1.30 \mathrm{a}$ & $1.07 \mathrm{a}$ \\
\hline \multicolumn{14}{|c|}{ Means for tractor passes } \\
\hline & P0 & 0.36 & 0.20 & $0.22 \mathrm{a}$ & 1.16 & 0.66 & 0.58 & 1.27 & 0.88 & $0.84 \mathrm{a}$ & 1.83 & $1.22 \mathrm{a}$ & $0.93 \mathrm{a}$ \\
\hline & P2 & 0.37 & 0.20 & $0.20 \mathrm{ab}$ & 1.24 & 0.74 & 0.56 & 1.26 & 0.94 & $0.75 \mathrm{ab}$ & 1.99 & $1.11 \mathrm{ab}$ & $0.94 \mathrm{a}$ \\
\hline & P4 & 0.39 & 0.20 & $0.17 \mathrm{ab}$ & 1.18 & 0.76 & 0.56 & 1.25 & 0.91 & $0.76 \mathrm{ab}$ & 1.88 & $0.98 \mathrm{ab}$ & $0.82 \mathrm{ab}$ \\
\hline & P6 & 0.37 & 0.19 & $0.15 \mathrm{~b}$ & 1.20 & 0.68 & 0.47 & 1.19 & 0.89 & $0.68 \mathrm{~b}$ & 1.83 & $0.95 \mathrm{~b}$ & $0.75 \mathrm{~b}$ \\
\hline
\end{tabular}

Explanations under Table 2

Table 5. Effect of tractor traffic and nitrogen fertilization on herbage yield ( $\left.\mathrm{t} \mathrm{ha}^{-1} \mathrm{DM}\right)$ of Phleum pratense

\begin{tabular}{|c|c|c|c|c|c|c|c|c|c|c|c|c|c|}
\hline \multirow{2}{*}{\multicolumn{2}{|c|}{ Treatments }} & \multicolumn{3}{|c|}{2009} & \multicolumn{3}{|c|}{2010} & \multicolumn{3}{|c|}{2011} & \multicolumn{3}{|c|}{2012} \\
\hline & & $1^{\text {st }}$ cut & $2^{\text {nd }}$ cut & $3^{\text {rd }}$ cut & $1^{\text {st }}$ cut & $2^{\text {nd }}$ cut & $3^{\text {rd }}$ cut & $1^{\text {st }}$ cut & $2^{\text {nd }}$ cut & $3^{\text {rd }}$ cut & $1^{\text {st }}$ cut & $2^{\text {nd }}$ cut & $3^{\text {rd }}$ cut \\
\hline \multirow{4}{*}{ No } & P0 & 0.57 & 0.31 & 0.30 & 0.35 & 0.24 & 0.23 & 0.41 & 0.30 & 0.29 & 0.18 & 0.17 & 0.14 \\
\hline & P2 & 0.58 & 0.30 & 0.28 & 0.37 & 0.29 & 0.24 & 0.41 & 0.34 & 0.26 & 0.18 & 0.16 & 0.13 \\
\hline & P4 & 0.62 & 0.31 & 0.25 & 0.40 & 0.34 & 0.26 & 0.39 & 0.36 & 0.26 & 0.19 & 0.12 & 0.14 \\
\hline & P6 & 0.61 & 0.29 & 0.19 & 0.35 & 0.32 & 0.22 & 0.41 & 0.31 & 0.25 & 0.19 & 0.10 & 0.12 \\
\hline \multirow{4}{*}{ N80 } & P0 & 0.71 & 0.41 & 0.44 & 0.64 & 0.33 & 0.31 & 0.45 & 0.32 & 0.31 & 0.43 & 0.26 & 0.20 \\
\hline & P2 & 0.80 & 0.44 & 0.42 & 0.68 & 0.37 & 0.25 & 0.43 & 0.33 & 0.28 & 0.48 & 0.25 & 0.20 \\
\hline & P4 & 0.81 & 0.42 & 0.37 & 0.60 & 0.36 & 0.26 & 0.42 & 0.30 & 0.27 & 0.40 & 0.19 & 0.15 \\
\hline & P6 & 0.79 & 0.40 & 0.30 & 0.65 & 0.33 & 0.22 & 0.40 & 0.31 & 0.24 & 0.39 & 0.19 & 0.16 \\
\hline \multirow{4}{*}{ N160 } & P0 & 0.85 & 0.50 & 0.57 & 0.75 & 0.43 & 0.33 & 0.55 & 0.37 & 0.34 & 0.50 & 0.27 & 0.20 \\
\hline & $\mathrm{P} 2$ & 0.85 & 0.48 & 0.50 & 0.80 & 0.45 & 0.35 & 0.56 & 0.38 & 0.30 & 0.53 & 0.24 & 0.23 \\
\hline & P4 & 0.88 & 0.45 & 0.38 & 0.77 & 0.45 & 0.32 & 0.57 & 0.37 & 0.32 & 0.46 & 0.23 & 0.20 \\
\hline & P6 & 0.85 & 0.44 & 0.40 & 0.80 & 0.38 & 0.27 & 0.51 & 0.37 & 0.28 & 0.48 & 0.24 & 0.16 \\
\hline \multicolumn{14}{|c|}{ Means for fertilization } \\
\hline & N0 & $0.60 \mathrm{~b}$ & $0.30 \mathrm{~b}$ & $0.25 \mathrm{~b}$ & $0.37 \mathrm{~b}$ & $0.30 \mathrm{~b}$ & $0.24 \mathrm{~b}$ & $0.40 \mathrm{~b}$ & 0.33 & 0.27 & $0.19 \mathrm{~b}$ & 0.14 & 0.13 \\
\hline & N80 & $0.77 \mathrm{ab}$ & $0.42 \mathrm{a}$ & $0.38 \mathrm{ab}$ & $0.64 \mathrm{ab}$ & $0.35 \mathrm{ab}$ & $0.26 \mathrm{ab}$ & $0.42 \mathrm{ab}$ & 0.31 & 0.27 & $0.43 \mathrm{a}$ & 0.22 & 0.18 \\
\hline & N160 & $0.86 \mathrm{a}$ & $0.47 \mathrm{a}$ & $0.46 \mathrm{a}$ & $0.78 \mathrm{a}$ & $0.43 \mathrm{a}$ & $0.32 \mathrm{a}$ & $0.55 \mathrm{a}$ & 0.37 & 0.31 & $0.49 \mathrm{a}$ & 0.25 & 0.20 \\
\hline \multicolumn{14}{|c|}{ Means for tractor passes } \\
\hline & $\mathrm{P} 0$ & 0.71 & 0.40 & $0.44 \mathrm{a}$ & 0.58 & 0.33 & 0.29 & 0.47 & 0.33 & 0.31 & 0.37 & 0.23 & 0.18 \\
\hline & $\mathrm{P} 2$ & 0.74 & 0.41 & $0.40 \mathrm{ab}$ & 0.62 & 0.37 & 0.28 & 0.46 & 0.35 & 0.28 & 0.40 & 0.21 & 0.19 \\
\hline & $\mathrm{P} 4$ & 0.77 & 0.39 & $0.33 \mathrm{ab}$ & 0.59 & 0.38 & 0.28 & 0.46 & 0.34 & 0.28 & 0.35 & 0.18 & 0.16 \\
\hline & $\mathrm{P} 6$ & 0.75 & 0.38 & $0.30 \mathrm{~b}$ & 0.60 & 0.34 & 0.24 & 0.44 & 0.33 & 0.26 & 0.35 & 0.18 & 0.15 \\
\hline
\end{tabular}

Explanations under Table 2 
Table 6. Effect of tractor traffic and nitrogen fertilization on herbage yield $\left(\mathrm{t} \mathrm{ha}{ }^{-1} \mathrm{DM}\right)$ of Poa pratensis

\begin{tabular}{|c|c|c|c|c|c|c|c|c|c|c|c|c|c|}
\hline \multirow{2}{*}{\multicolumn{2}{|c|}{ Treatments }} & \multicolumn{3}{|c|}{2009} & \multicolumn{3}{|c|}{2010} & \multicolumn{3}{|c|}{2011} & \multicolumn{3}{|c|}{2012} \\
\hline & & $1^{\text {st }}$ cut & $2^{\text {nd }}$ cut & $3^{\text {rd }}$ cut & $1^{\text {st }}$ cut & $2^{\text {nd }}$ cut & $3^{\text {rd }}$ cut & $1^{\text {st }}$ cut & $2^{\text {nd }}$ cut & $3^{\text {rd }}$ cut & $1^{\text {st }} \mathrm{cut}$ & $2^{\text {nd }}$ cut & $3^{\text {rd }}$ cut \\
\hline \multirow{4}{*}{ N0 } & P0 & 0.29 & 0.15 & 0.15 & 0.17 & 0.12 & 0.12 & 0.41 & 0.30 & 0.29 & 0.39 & 0.37 & 0.27 \\
\hline & P2 & 0.29 & 0.15 & 0.14 & 0.19 & 0.15 & 0.12 & 0.41 & 0.34 & 0.26 & 0.39 & 0.31 & 0.25 \\
\hline & P4 & 0.31 & 0.15 & 0.12 & 0.20 & 0.17 & 0.13 & 0.39 & 0.36 & 0.26 & 0.45 & 0.29 & 0.28 \\
\hline & P6 & 0.31 & 0.14 & 0.10 & 0.17 & 0.16 & 0.11 & 0.41 & 0.31 & 0.25 & 0.43 & 0.25 & 0.25 \\
\hline \multirow{4}{*}{ N80 } & P0 & 0.35 & 0.20 & 0.22 & 0.32 & 0.16 & 0.15 & 0.45 & 0.32 & 0.31 & 0.80 & 0.51 & 0.45 \\
\hline & P2 & 0.40 & 0.22 & 0.21 & 0.34 & 0.18 & 0.13 & 0.43 & 0.33 & 0.28 & 0.78 & 0.49 & 0.38 \\
\hline & P4 & 0.40 & 0.21 & 0.18 & 0.30 & 0.18 & 0.13 & 0.42 & 0.30 & 0.27 & 0.79 & 0.37 & 0.33 \\
\hline & P6 & 0.39 & 0.20 & 0.15 & 0.33 & 0.17 & 0.11 & 0.40 & 0.31 & 0.24 & 0.78 & 0.41 & 0.31 \\
\hline \multirow{4}{*}{ N160 } & P0 & 0.43 & 0.25 & 0.28 & 0.38 & 0.21 & 0.16 & 0.55 & 0.37 & 0.34 & 0.62 & 0.45 & 0.35 \\
\hline & P2 & 0.42 & 0.24 & 0.25 & 0.40 & 0.23 & 0.17 & 0.56 & 0.38 & 0.30 & 0.67 & 0.39 & 0.40 \\
\hline & P4 & 0.44 & 0.23 & 0.19 & 0.38 & 0.22 & 0.16 & 0.57 & 0.37 & 0.32 & 0.76 & 0.36 & 0.33 \\
\hline & P6 & 0.42 & 0.22 & 0.20 & 0.40 & 0.19 & 0.14 & 0.51 & 0.37 & 0.28 & 0.72 & 0.32 & 0.28 \\
\hline \multicolumn{14}{|c|}{ Means for fertilization } \\
\hline & N0 & $0.30 \mathrm{~b}$ & $0.15 \mathrm{~b}$ & $0.13 \mathrm{~b}$ & $0.18 \mathrm{~b}$ & $0.15 \mathrm{~b}$ & 0.12 & 0.40 & 0.33 & 0.27 & 0.42 & 0.30 & 0.26 \\
\hline & N80 & $0.39 \mathrm{ab}$ & $0.21 \mathrm{a}$ & $0.19 \mathrm{ab}$ & $0.32 \mathrm{ab}$ & $0.17 \mathrm{ab}$ & 0.13 & 0.42 & 0.31 & 0.27 & 0.79 & 0.44 & 0.37 \\
\hline & N160 & $0.43 \mathrm{a}$ & $0.23 \mathrm{a}$ & $0.23 \mathrm{a}$ & $0.39 \mathrm{a}$ & $0.21 \mathrm{a}$ & 0.16 & 0.55 & 0.37 & 0.31 & 0.69 & 0.38 & 0.34 \\
\hline \multicolumn{14}{|c|}{ Means for tractor passes } \\
\hline & P0 & 0.36 & 0.20 & $0.22 \mathrm{a}$ & 0.29 & 0.17 & 0.14 & 0.47 & 0.33 & 0.31 & 0.60 & 0.44 & 0.35 \\
\hline & P2 & 0.37 & 0.20 & $0.20 \mathrm{ab}$ & 0.31 & 0.19 & 0.14 & 0.46 & 0.35 & 0.28 & 0.62 & 0.40 & 0.34 \\
\hline & P4 & 0.39 & 0.20 & $0.17 \mathrm{ab}$ & 0.29 & 0.19 & 0.14 & 0.46 & 0.34 & 0.28 & 0.67 & 0.34 & 0.31 \\
\hline & P6 & 0.37 & 0.19 & $0.15 \mathrm{~b}$ & 0.30 & 0.17 & 0.12 & 0.44 & 0.33 & 0.26 & 0.64 & 0.33 & 0.28 \\
\hline
\end{tabular}

Explanations under Table 2

to tractor traffic mainly during the third cut. However, this effect on annual production of grass species was not very evident. A significant difference was noticed only for $L$. perenne. For all investigated components of the mixture no interaction between treatments, tractor traffic and $\mathrm{N}$ fertilization was observed.

\section{Discussion}

It has been widely recognized that nitrogen fertilization increased grassland productivity (Klimek et al., 2007; van Eekeren et al., 2010). All investigated grasses increased their biomass production as a reaction to higher nitrogen fertilization. According to Leps (1999), nitrogen fertilization usually plays an advantageous role in the growing conditions of tall grasses like $F$. pratensis, $P$. pratensis and Agropyron repens. Short grasses are usually displaced by tall ones. In this research some small grasses like Poa annua disappeared in the third year of the experiment.

One of the most important components in the botanical composition of grasslands is legumes. However, the nitrogen fertilization used to increase dry matter yield usually results in a decrease in legume ratios in the botanical composition (Koc, 2013). In this experiment legumes were represented by T. pratense. As expected, this species slowly disappeared when mineral fertilization was applied. In the second year of the experiment $T$. pratensis was absent at the object with $\mathrm{N}$ rates of $160 \mathrm{~kg} \mathrm{ha}^{-1}$. It persisted at the N0 (nonfertilized) treatment, up to 2012. In the previous author's research (Głąb, Kacorzyk, 2011), the presence of a similar relationship was confirmed for meadows located in mountain regions. Under higher $\mathrm{N}$ rates, mineral or organic, the participation of $T$. repens and small grasses decreased.

The results obtained in this field experiment indicated that tractor traffic of red clover/grass meadow significantly affected the botanical composition and herbage production of its components. As more intensive wheeling was applied, a decrease in plant yield was recorded. This relationship has been widely confirmed by other authors (Whalley et al., 2008). It is usually ascribed to unfavourable changes of soil properties. Soil compaction leads to soil structure degradation, which is strongly associated with changes in physical properties of soil like porosity, bulk density, and penetration resistance
(Krebstein et al., 2013; Nawaz et al., 2013). The degraded physical environment of the soil due to compaction influences mainly the growth and development of roots. Soil compaction increases mechanical impendence, creates unfavourable growing conditions for roots, and restricts oxygen, water and nutrients supply (Bhandral et al., 2007). Changes in physical properties resulted also in the biological activity of compacted soil. According to Tian et al. (2015), physical properties of soil affected the number and activity of microorganisms and their physiological diversity. Changes in biological activity resulted in processes catalyzed by microorganisms which can modify chemical properties of soil and availability of nutrients (Nosalewicz, Nosalewicz, 2011). It could be expected that reduced root system and the decreased nutrient availability in compacted soils caused lower nutrient uptake by the growing crop and hence decreased shoot growth and crop yield. Kristoffersen and Riley (2005) confirmed this relationship for phosphorus uptake by barley. The results of the current experiment also are based on this theory.

However, the reaction of root systems of perennial forage plans to soil compaction can vary and can be described as positive or negative. It was reported that a common response of the root system to increasing bulk density is to decrease its length and biomass in the upper soil layer (Chen, Weil, 2010). Also, previous researches conducted by the author (Głąb, 2013) confirmed this trend for meadow fescue (F. pratensis) and Kentucky bluegrass $(P$. pratensis). Sometimes, the positive correlation between soil density and root characteristics was observed, particularly for perennial crops. In the investigation with tall fescue (Festuca arundinacea Schreb.), the soil compaction increased the dry matter, length of roots and their diameter (Głąb, 2007). This effect can be attributed to the activity of dense perennial plant roots and earthworms and is usually responsible for favourable changes in the pore system.

Based on these statements, it can be concluded that the yield depression that is observed here is the result of not only deteriorated soil structure and limited root growth but also is combined with mechanical damage to the aboveground part of plants. This resistance to mechanical stress is usually ascribed to anatomical properties, particularly the area of sclerenchyma cells (Evans et al., 2007). 
The results of the current study lay greater emphasis on nitrogen level than intensity of tractor traffic. It can be stated that sometimes it is difficult to compare the proportion between these two factors, fertilization and traffic intensity, in different experiments because they depend on numerous biotic and abiotic parameters. What can influence the final results are soil parameters like water content, initial bulk density, tractor weight and unit pressure, botanical composition of sward, etc. However, the common conclusion from these experiments is that both, $\mathrm{N}$ fertilization and traffic intensity significantly affect perennial plant yields. The important question here is whether these factors act in combination with each other or act independent of one another.

Bingham et al. (2010) found no interaction between soil compaction and the $\mathrm{N}$ fertilization level in an investigation on young barley plants. It could be stated that these two factors seem to act independently. Bingham et al. (2010) also did not find a significant interaction between compaction and nitrogen supply on root growth and biomass production of barley in the experiment with the deficit nitrogen supply. It could be expected that soil compaction leads to limitation in nitrogen uptake by plants. It is widely recognized that soil compaction caused by tractor wheeling results in deterioration of soil structure and its pore system (Raper, 2005). The consequence is restricted aeration and increased denitrification losses of $\mathrm{N}-\mathrm{NO}_{3}$. Furthermore, it leads to inhibited root growth and decreases mineralization of organic $\mathrm{N}$ (Głą, 2013). All these events presumably result in decreasing fertilizer efficiency and hence biomass production. These relationships should result in significant interaction in plant yields between traffic intensity and the level of $\mathrm{N}$ fertilization. However, in this research there was no statistically significant evidence for such interaction.

\section{Conclusions}

1. Nitrogen fertilization significantly affected the percentage of only three species, Trifolium pratense, Lolium perenne and Festuca pratensis. Percentage of T. pratense decreased with increasing nitrogen rates. The share of $T$. pratense changed from $13.4 \%$ in the control (N0) treatment to $5.4 \%$ in the treatment with the rate of $160 \mathrm{~kg} \mathrm{ha}^{-1} \mathrm{~N}$. Phleum pratense and Poa pratensis were not affected by nitrogen fertilization. However, the participation of $T$. pratense in the sward increased when higher nitrogen rates were applied in the first year of the trial, whereas in the following years, the reaction was opposite. The percentage of $L$. perenne decreased in 2009 from $37.2 \%$ in the untreated control to $36.2 \%$ in the $160 \mathrm{~kg} \mathrm{ha}^{-1} \mathrm{~N}$ treatment. In the next year this species positively reacted to nitrogen fertilization.

2 . The tractor traffic significantly affected the participation of $T$. pratense and L. perenne in the sward biomass. The percentage of $T$. pratense was reduced by wheel traffic, and this space was occupied by L. perenne. At the noncompacted treatment, the share of $T$. pratense was $12.3 \%$ and decreased to $6.5 \%$ when six tractor passes were applied. Other tested grasses were not influenced by the tractor passes applied.

3. The annual biomass production of the tested mixture was affected by both experimental treatments, nitrogen fertilization and traffic intensity. The lower value for dry matter was obtained in the control (N0) treatment without nitrogen fertilization $-8.38 \mathrm{tha}^{-1} \mathrm{DM}$. The rate of $160 \mathrm{~kg} \mathrm{ha}^{-1} \mathrm{~N}$ increased biomass production up to $12.28 \mathrm{t} \mathrm{ha}^{-1} \mathrm{DM}$. As more intensive wheeling was applied, a decrease in plant yields was recorded. However, for some species, this effect differed between years.
4. The biomass production of the tested species was affected by nitrogen fertilization. However, this reaction changed with time. The dry matter productivity of $T$. pratense increase in the first year when higher rates of nitrogen were applied. However, in the following years, this species started to disappear due to treatments with higher nitrogen rates.

5. The most susceptible species on soil compaction was $T$. pratense. The grasses showed a negative reaction to tractor traffic mainly during the third cuts. However, this effect on annual production of grass species was not evident.

\section{Acknowledgements}

Financial support for this study was provided by the Ministry of Science and Higher Education in Poland (grant No. N N313 146738).

Received 08032016

Accepted 22062016

\section{References}

Baritaux V., Houdart M., Boutonnet J. P., Chazoule C., Corniaux C., Fleury P., Lacombe N., Napoléone M., Tourrand J. F. 2016. Ecological embeddedness in animal food systems (re-)localisation: a comparative analysis of initiatives in France. Morncco and Senegal. Inurnal of Rural Studies. 43: 13-26 http://dx.doi.org/10.1016/j.jrurstud.2015.11.009

Bhandral R., Saggar S., Bolan N. S., Hedley M. J. 2007. Transformation of nitrogen and nitrous oxide emission from grassland soils as affected by compaction. Soil and Tillage Research. 94: 482-492 http://dx.doi.org/10.1016/j.still.2006.10.006

Bingham 1. J., Bengough A. G., Rees R. M. 2010. Soil compaction- $\mathrm{N}$ interactions in barley: Root growth and tissue comnosition. Soil and Tillage Research, 106:241-246 http://dx.doi.org/10.1016/j.still.2009.10.008

Chen G., Weil R. R., 2010. Penetration of cover crop roots through comnacted soils. Plant and Soil 331: 31-43 http://dx.doi.org/10.1007/s11104-009-0223-7

COM(2006)231 - EUR-Lex - Europa. Communication from the Commission to the Council, the European Parliament, the European Economic and Social Committee and the Committee of the Regions. Thematic strategy for soil protection. Brussels, Belgium

Evans L. S., Kahn-Jetter Z., Marks C., Harmoney K. R. 2007. Mechanical properties and anatomical components of stems of 42 grass species. Iournal of the Torrey Botanical Society, 134: 458-467 http://dx.doi.org/10.3159/07-RA-009.1

Gaujour E., Amiaud B., Mignolet C., Plantureux S. 2012. Factors and processes affecting plant biodiversity in permanent grasslands. A review. Agronomy for Sustainable Develonment. 32 (1): 133-160 http://dx.doi.org/10.1007/s13593-011-0015-3

Głab I. 2007. Etfect of soil compaction on root system development and yields of tall fescue. International Agrophysics, 21: 233-239

Głąb T. 2008. Effects of tractor wheeling on root morphology and yield of Lucerne, Medicago sativa L. Grass and Forage Science 63: 398-406 http://dx.doi.org/10.1111/j.1365-2494.2008.00647.x

Głąb T. 2013. Impact of soil compaction on root development and yield of meadow-grass. International Agrophysics, 27: 7-13

Głąb T., Kacorzyk P. 2011. Root distribution and herbage production under different management regimes of mountain grassland. Soil and Tillage Research. 113:99-104 http://dx.doi.org/10.1016/j.still.2011.02.008

Klimek S., Kemmermann A. R., Hotmann M., Isselstein J. 2007. Plant species richness and composition in managed grasslands: The relative importance of field management and environmental factors. Biolngical Conservation. 134: 559-570 http://dx.doi.org/10.1016/j.biocon.2006.09.007

Koc A. 2013. Effect of phosphorus doses and application time on the yield and quality of hay and botanical composition of clover dominant meadow in highlands of Turkey. Turkish Journal of Field Crops, 18 (2): 205-210 
Krebstein K., von Janowsky K., Reintam E., Horn R., Leeduks J., Kuht J. 2013. Soil compaction in a Cambisol under grassland in Fstonia. 7emdirhyste-Agriculture, 100 (1): 33-38 http://dx.doi.org/10.13080/z-a.2013.100.005

Kristoffersen A. K., Riley H. 2005. Etfects of soil compaction and moisture regime on the root and shoot growth and phosphorus uptake of barley plants growing on soils with varying phosphorus status. Nutrient Cycling in Agroecosvstems. 72: 135-146 http://dx.doi.org/10.1007/s10705-005-0240-8

Leps J. 1999. Nutrient status, disturbance and competition: an experimental test of relationships in a wet meadow. Journal of Vegetation Science, 10: 219-230 http://dx.doi.org/10.2307/3237143

Marini L., Scotton M., Klimek S., Isselstein J., Pecile A. 2007. Effects of local factors on plant species richness and composition of Alpine meadows. Agriculture, Ecosystems and Environment. 119: 281-288 http://dx.doi.org/10.1016/j.agee.2006.07.015

Nawaz M. F., Bourrie G., Irolard F. 2013. Soil compaction impact and modelling. A review. Agronomy for Sustainable Develonment, 33: 291-309 http://dx.doi.org/10.1007/s13593-011-0071-8

Newell-Price J. P., Whittingham M. J., Chambers B. J., Peel S. 2013. Visual soil evaluation in relation to measured soil physical properties in a survey of grassland soil compaction in Fngland and Wales. Soil and Tillage Research, 127: 65-73 http://dx.doi.org/10.1016/j.still.2012.03.003

Nosalewicz A., Nosalewicz M. 2011. Etfect of soil compaction on dehydrogenase activity in bulk soil and rhizosphere. International Agrophysics, 25: 47-51

Raper R. L. 2005. Agricultural traffic impacts on soil. Journal of Terramechanics, 42: 259-280

http://dx.doi.org/10.1016/j.jterra.2004.10.010
Scott D. I., Tams A. R., Berry P. M., Mooney S. J. 2005. The effects of wheel-induced soil compaction on anchorage strength and resistance to root lodging of winter barley, Hordeum vulgare L. Soil and Tillage Research, 82: 147-160 http://dx.doi.org/10.1016/j.still.2004.06.008

State of Application of Regulation (EEC) 2078/92. DG VI/7655/98 Commission Working Document. Evaluation of agri-environment programmes

Tephnadze N., Abdaladze O., Nakhutsrishvili G., Simmering D., Waldhardt R., Otte A. 2014. The impacts of management and site conditions on the phytodiversity of the upper montane and subalpine belts in the Central Greater Caucasus. Phytocoenologia, 44 (3-4): 255-291 http://dx.doi.org/10.1127/0340-269X/2014/0044-0579

Tian W., Wang L., Li Y., Zhuang K., Li G., Zhang J., Xiao X., Xi Y. 2015. Responses of microbial activity, abundance, and community in wheat soil after three years of heavy fertilization with manure-based compost and inorganic nitrogen. Agriculture, Fcosystems and Fnvironment, 213: 219-227 http://dx.doi.org/10.1016/j.agee.2015.08.009

van Eekeren N., de Boer H., Hanegraat M., Bokhorst J., Nierop D., Bloem J., Schouten T., de Goede R., Brussaard L. 2010. Ecosystem services in grassland associated with biotic and abiotic soil parameters. Soil Biology and Biochemistry, 42: 1491-1504 http://dx.doi.org/10.1016/j. soilbio.2010.05.016

Whalley W. R., Watts C. W., Gregory A. S., Mooney S. J., Clark L. J., Whitmore A. P. 2008. The effect of soil strength on the vield of wheat. Plant and Soil. 306: 237-247 http://dx.doi.org/10.1007/s11104-008-9577-5

WRB 2014. World reference base for soil resources 2014 International soil classification system for naming soils and creating legends for soil maps. World Soil Resources Reports No. 106. FAO, Rome

ISSN 1392-3196 / e-ISSN 2335-8947

Zemdirbyste-Agriculture, vol. 103, No. 4 (2016), p. 347-354

DOI $10.13080 / \mathrm{z}-\mathrm{a} .2016 .103 .044$

\title{
Dirvų išvažinẻjimas ir tręšimas azotu veikia žolynų produktyvumą
}

\author{
T. Glab, A. Żabinski, U. Sadowska
}

Krokuvos žemès ūkio universiteto Technikos eksploatacijos, ergonomikos ir gamybos procesų institutas, Lenkija

\section{Santrauka}

Pastaraisiais metais žemės ūkio technikos sukeltas dirvožemio suslègimas yra laikomas vienu veiksnių, mažinančių daugiamečių augalų derlių. Kartu vyksta ir žolynų tręšimo intensyvumo pokyčiai. Šiuo tyrimu siekta įvertinti sąveiką tarp ịvairių tręšimo azotu normų ir išvažinèjimo intensyvumo. Lauko bandymas atliktas 2009-2012 m. smèlingo priemolio dirvožemyje (Mollic Fluvisol) Mydlniki vietovejje netoli Krokuvos, Lenkijoje. Bandymas atliktas taikant skaidytų laukelių metodą keturiais pakartojimais. Tręštas pagrindinis laukelis, o dirvos išvažinėjimas tirtas daliniame laukelyje. Bandymo metu azotu tręšta trimis lygiais: netręšta (kontrolinis variantas), tręšta 80 bei $160 \mathrm{~kg} \mathrm{ha}^{-1} \mathrm{~N}$, ir tirti keturi išvažinejjimo traktoriumi intensyvumo lygiai: be važiavimų (kontrolinis variantas), du, keturi ir šeši važiavimai.

Tręšimas azotu smarkiai paveikè tik trijų rūšių augalų - Trifolium pratense, Lolium perenne ir Festuca pratensis - procentą žolyne. Didesnès normos azoto turèjo neigiamos įtakos T. pratense, o L. perenne ir F. pratensis priešingai. Dirvos išvažinèjimas smarkiai paveikè T. pratense ir L. perenne išplitimą žolyne. Dèl žolyno išvažinèjimo T. pratense procentas sumažèjo, o išnykusių augalų vietą užèmè $L$. perenne. Botaninèse sudèties pokyčiai lèmė žolyno produktyvumą. Visų tirtų rūšių augalų biomasės produktyvumas padidèjo proporcingai azoto normoms. Tręšimas azotu neigiamai paveikè tik T. pratense, ir nuo $2011 \mathrm{~m}$. jie pradèjo nykti žolynuose, tręštose didesnèmis normomis azoto. Visų tirtų mišinio komponentų nebuvo nustatyta sąveika tarp variantų, dirvų išvažinèjimo ir tręšimo azotu.

Reikšminiai žodžiai: botaninė sudètis, raudonieji dobilai, tręšimas azotu, žolynai, žolynų produktyvumas.

Please use the following format when citing the article:

Glab T., Żabinski A., Sadowska U. Tractor traffic and nitrogen fertilization affect the herbage production of the red clover/grass sward. Zemdirbyste-Agriculture, 103 (4): 347-354 DOI 10.13080/z-a.2016.103.044 